\title{
Gamma Ray Measurement Information Barriers for the FMTT Demonstration System
}

J. K. Wolford, Jr.

\section{October 1, 2000}

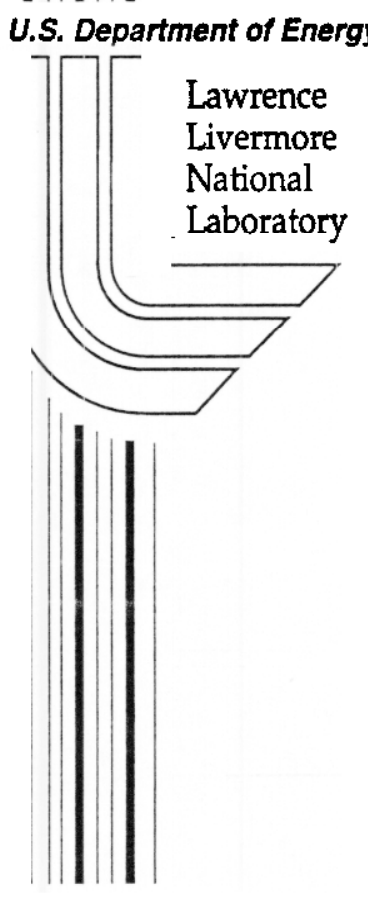




\section{DISCLAIMER}

This document was prepared as an account of work sponsored by an agency of the United States Government. Neither the United States Government nor the University of California nor any of their employees, makes any warranty, express or implied, or assumes any legal liability or responsibility for the accuracy, completeness, or usefulness of any information, apparatus, product, or process disclosed, or represents that its use would not infringe privately owned rights. Reference herein to any specific commercial product, process, or service by trade name, trademark, manufacturer, or otherwise, does not necessarily constitute or imply its endorsement, recommendation, or favoring by the United States Government or the University of California. The views and opinions of authors expressed herein do not necessarily state or reflect those of the United States Government or the University of California, and shall not be used for advertising or product endorsement purposes.

Work performed under the auspices of the U.S. Department of Energy by the University of California Lawrence Livermore National Laboratory under Contract W-7405-Eng-48.

This report has been reproduced directly from the best available copy.

Available to DOE and DOE contractors from the Office of Scientific and Technical Information

P.O. Box 62, Oak Ridge, TN 37831

Prices available from (423) 576-8401

http://apollo.osti.gov/bridge/

Available to the public from the

National Technical Information Service

U.S. Department of Commerce 5285 Port Royal Rd., Springfield, VA 22161

http://www.ntis.gov/

OR

Lawrence Livermore National Laboratory Technical Information Department's Digital Library

http://www.llnl.gov/tid/Library.html 


\title{
Gamma Ray Measurement Information Barriers for the FMTT Demonstration System
}

\author{
Fissile Material Transparency Technology Demonstration, Los Alamos \\ National Laboratory, August 16, 2000.
}

James K. Wolford, Jr., Lawrence Livermore National Laboratory

\section{Introduction and Scope}

The gamma ray attribute measurement information barrier discussion directly complements the discussion of gamma ray measurement, presented in the measurements paper by Gosnell and the general discussion of information barriers (IBs) by MacArthur. It focuses on the information barrier features applied specifically to the gamma-ray measurement and attribute analysis system. The FMTT demonstration instrument represents the second application of an IB design paradigm developed in conjunction with the Joint DOE/DoD Information Barriers Working Group (IBWG) as well as representatives from the Russian Federation's delegations to the Trilateral Initiative and meetings on the agreement for transparency at the Mayak Fissile Storage Facility (FMSF). It is also the second evolutionary step in constructing hardware to embody these jointly developed ideas. The first step was the prototype instrument developed for the Trilateral Initiative, the so-called Attribute Verification System with Information Barriers for Plutonium with Classified Characteristics utilizing Neutron Multiplicity Counting and High-Resolution Gamma-ray Spectroscopy (AVNG), that was demonstrated at Los Alamos National Laboratory in June 1999. Several improvements are evident in this second effort, and will be discussed below. Improved, though this information barrier may be, it is still a prototype meant only for demonstration purposes. Its evolving specification and design are appropriately a subject for joint discussion and development. Part of that development must include creating components that the respective governments can trust enough to certify.

\section{System Overview}

The illustration shows an overview of the gamma-ray attribute measurement system, including one of the two gamma-ray detectors to be used in this demonstration. Features of the information barrier are present in each of the elements shown, and the remainder of this commentary will them in some detail. To summarize these features, they include:

- Restricted energy windows which limit the range of gamma-ray spectrum data present in the computers that derive the attributes.

- Reduced functionality (single board) computer and its custom software.

- Tungsten iris

- RF shielding surrounding the detector, detector pre-amp, tungsten iris, and iris controller. 


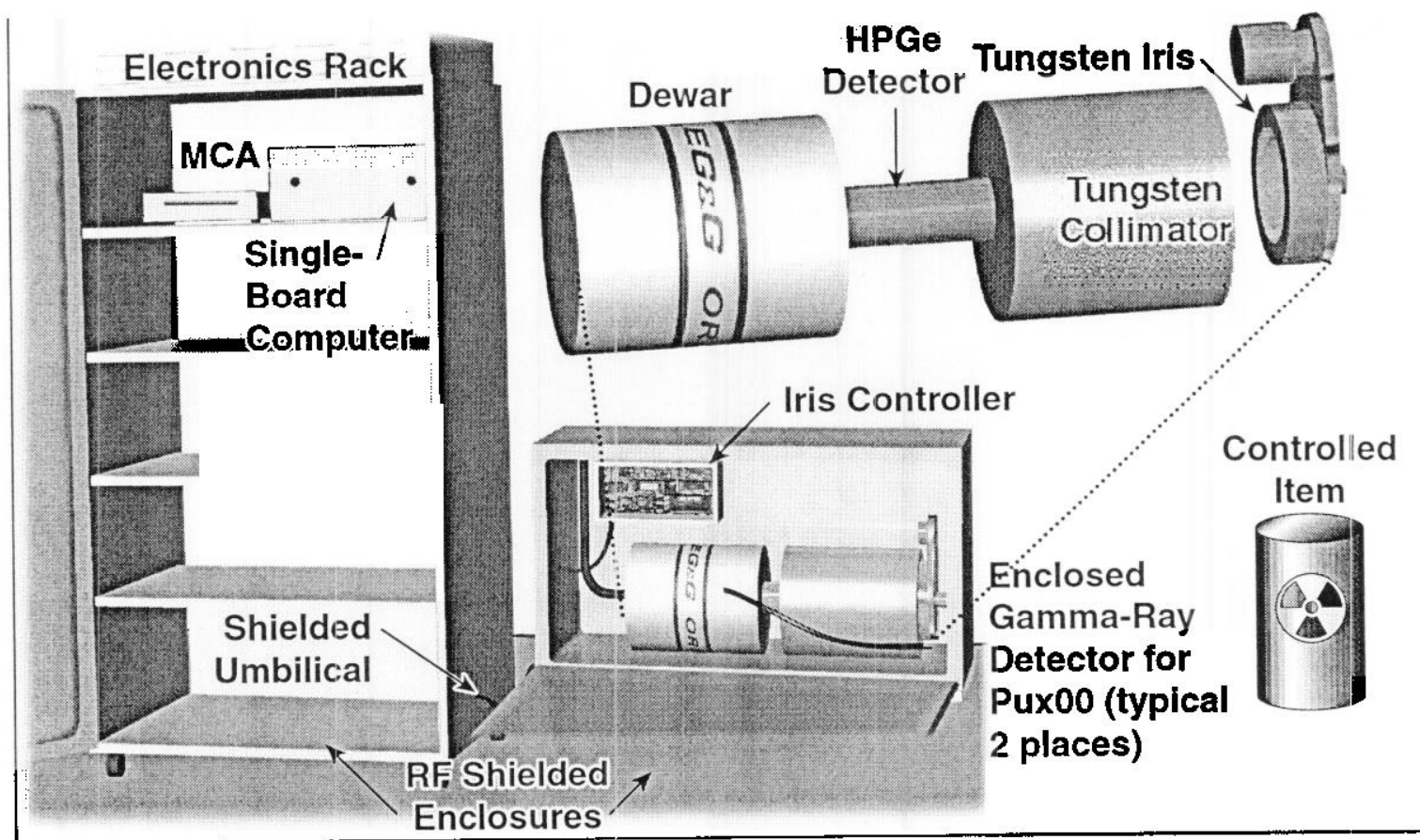

Figure 1. Overview of the information barrier for the FMTT demonstration gamma-ray measurements, showing one of the two gamma-ray detection systems used to collect data and derive the required attributes.

\section{System Design Criteria Related to the Information Barrier}

From the very start of the instrument design process, planners kept in mind the goals of the information barrier. To recapitulate Duncan MacArthur's overview statement, the first goal of the information barrier is to "allow meaningful measurements while preventing the release of classified information". To meet this goal, the gamma-ray detector and analyzer assemblies have been designed and built to minimize the data they collect, protect the data as it is transferred between elements, and output only the simple unclassified results that the monitor requires. The next section summarizes these design features.

\section{Information Barrier Related Design Features}

The design of the information barrier reflects shared U.S. and Russian Federation concerns about the protection of classified information. The specific design features which will be described in detail below include:

- Restriction of primitive data (portions of the gamma-ray spectrum) collected to that which is absolutely required for the attribute calculations.

- Surrounding of both the detectors and the analyzers by RF shielding.

- The gamma-ray flux is restricted by two measures. First the system acquires data for a fixed interval (wall clock time) which is somewhat greater than that needed to derive accurate attributes. Second, the front face of the detector is covered by an autoadjusting iris, which opens and closes to optimize the live time of the measurement. The instantaneous size of the aperture is obscured by the enclosure that prevents guessing at the gamma ray dose.

- Commercial components were used wherever possible to increase reliability and "familiarity." The importance of this may not be obvious, however using commercial 
components takes advantage of the vendor's quality control testing and product refinement efforts and simplifies eventual inspection efforts by presenting the inspector with a system that should be identical to others that are easily obtainable.

- The computers that acquired the data and reduce it to attributes have been simplified to remove unnecessary functionality. They service a minimum of peripherals and contain no persistent memory. They contain a small number of circuit elements, which should also simplified.

\section{Restricted Spectrum Windows}

Figure 2, which was presented in Gosnell's talk on gamma-ray measurements, is repeated below because it also illustrates an information barrier feature. One very direct way of reducing the amount of classified information present in the system is to filter out everything that is not required, at the acquisition stage. As the insets in the diagrams below suggest, the attribute calculations require as input only very narrow windows of the spectrum. The energy dependence of the spectrum intensities shows directly the advantage of performing parallel measurements using two detectors. The measurement above $800 \mathrm{keV}$ will obviously take more time and justifies a dedicated detector system. This distributes the computational load and reduces the amount of classified data that must be retained in memory between measurements.

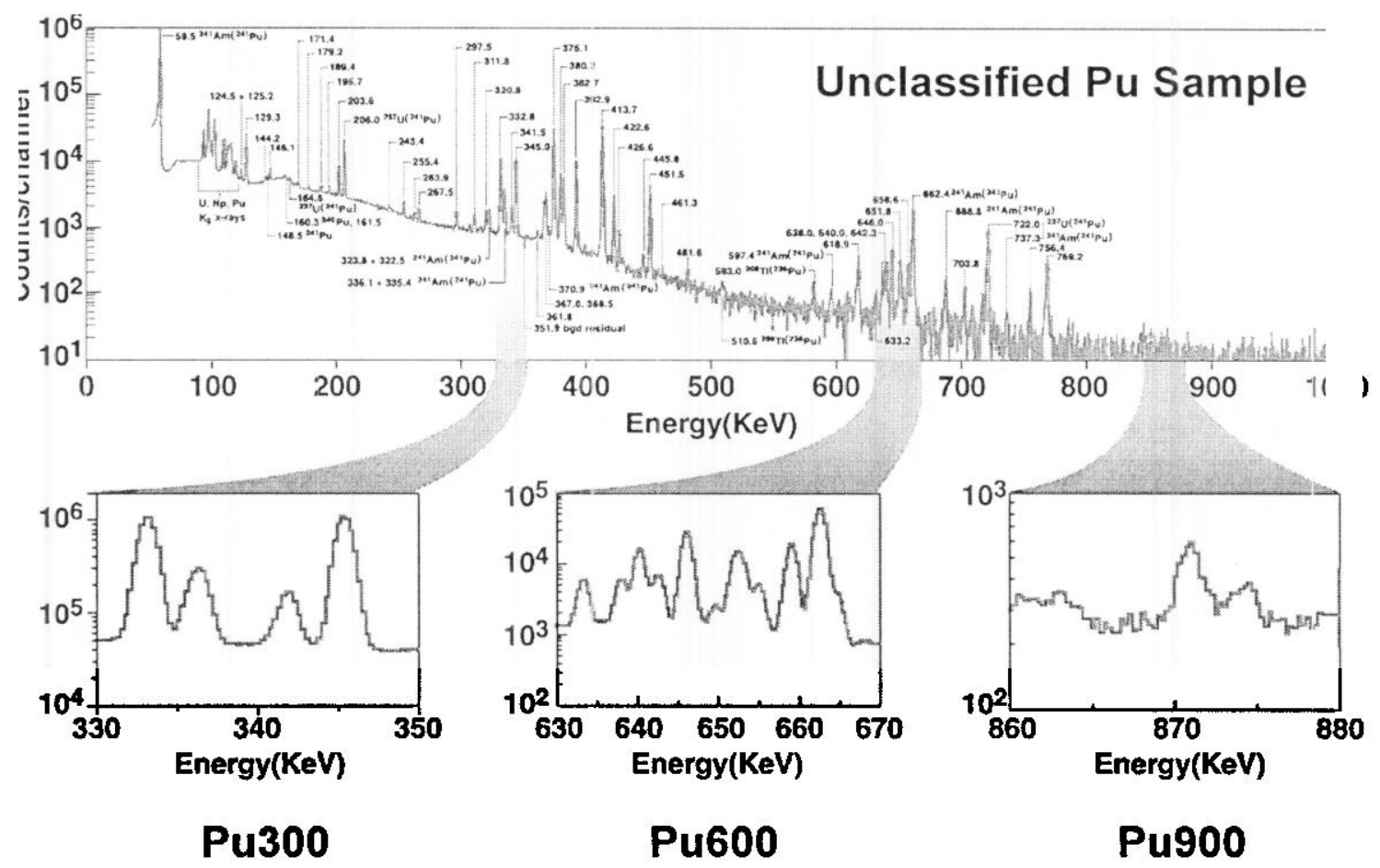

gure 2. Gamma ray spectrum from an unclassified source showing detail of the portions actually used to srive the attributes. The Pu300 calculation provides information on Pu presence and "age", Pu600 provides additional information on presence and isotopic ratio, while Pu900 indicates the existence of oxide.

\section{RF Shielded Enclosures}

The data signals present in a gamma-ray spectroscopy system include fast rising analog pulses and digital switching waveforms, both of which produce broadband radio 
frequency emanations. To prevent the broadcasting of classified information in the system, we have placed both the gamma ray detectors and their associated electronics inside of shielded conducting enclosures that are carefully grounded. As provided by commercial suppliers, these enclosures meet exacting standards for attenuation over the relevant frequency range. The cables connected to them are also shielded and grounded. And the system as shown in figure 3 would lend itself to additional shielding as the respective governments might require.

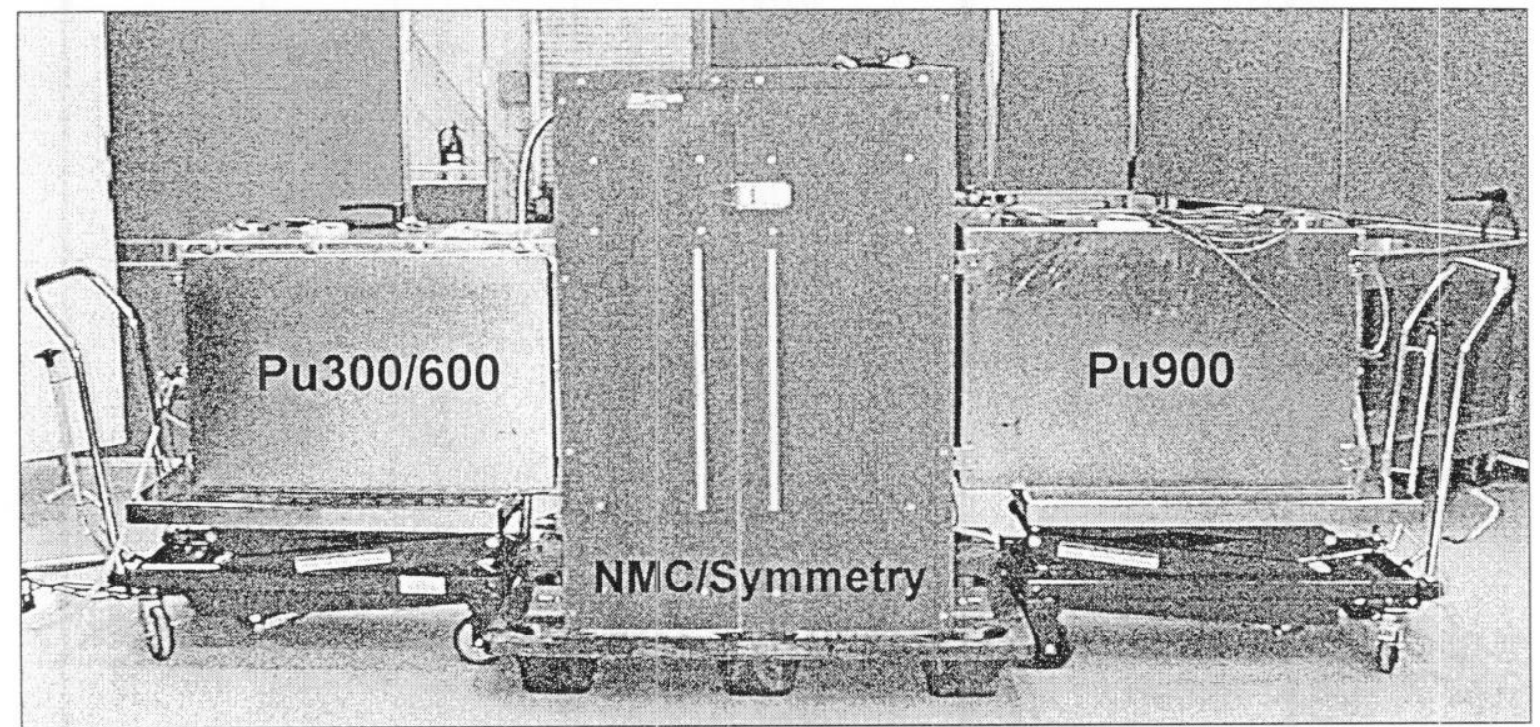

Figure 3. Gamma-ray detector assemblies shown inside RF-shielded enclosures adjacent to neutron multiplicity counter. The enclosures are secured and configured to measure a classified component. This arrangement allows simultaneous measurement of a classified item inside the NMC. Opening either the $\mathrm{Pu} 300 / 600$ or the Pu900 enclosure during a classified measurement will signal the security watchdog (not shown) to withdraw power from the entire measurement system.

\section{Gamma-ray Detection Systems Inside the Shielded Enclosures}

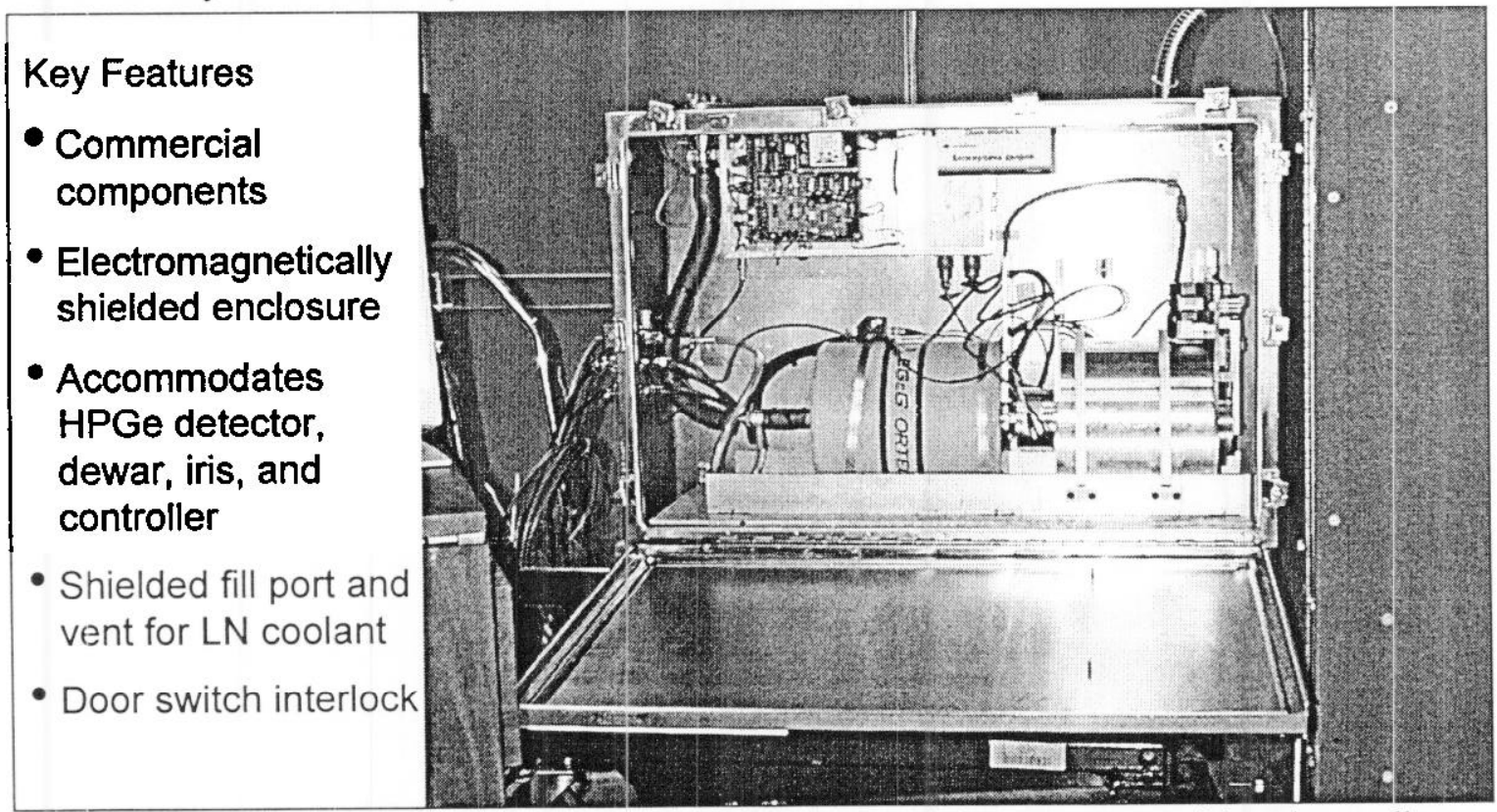

Figure 4. View inside the shielded enclosure housing the Pu300/600 detector, tungsten iris and controller. 
Figure 4 shows a view inside one of the two shielded enclosures housing the gamma-ray detectors. For the most part, the system consists of commercial components, including the enclosure itself. Exceptions include the annular tungsten shield surrounding the detector, and the tungsten iris in front of it. The enclosure was chosen to be large enough to accommodate the detector, its liquid nitrogen dewar and fill tube, the tungsten shield and the iris, along with its controller unit. The dewar has enough capacity to keep the detector cool for at least 24 hours, and the vented fill system permits the dewar to be refilled without opening the door. When the door is opened, an interlock (mounted in the top center of the lip of the door opening) signals back to the security watchdog that the system has been breached. If the interlock opens during a classified measurement, the watchdog will effect an immediate power shutdown.

\section{Reduced Functionality Computer (Attribute Analyzer)}

The attribute analyzers consist of single board computers designed so that the information they contain is localized and short-lived. They are composed entirely of commercial parts that are very common and inexpensive. Their computational engines are Intel 80486 microprocessors, which are well matched to the computational load. They are packaged as modules on printed circuit cards which are slightly over $8 \mathrm{~cm}$ on a side. When more than one card is present in a system, they communicate over an industry standard interface (PC-104). For this demonstration, they are installed in protective enclosures along with their power supplies and port connectors. Figure 5 shows one of the analyzer ınits with its cover removed.

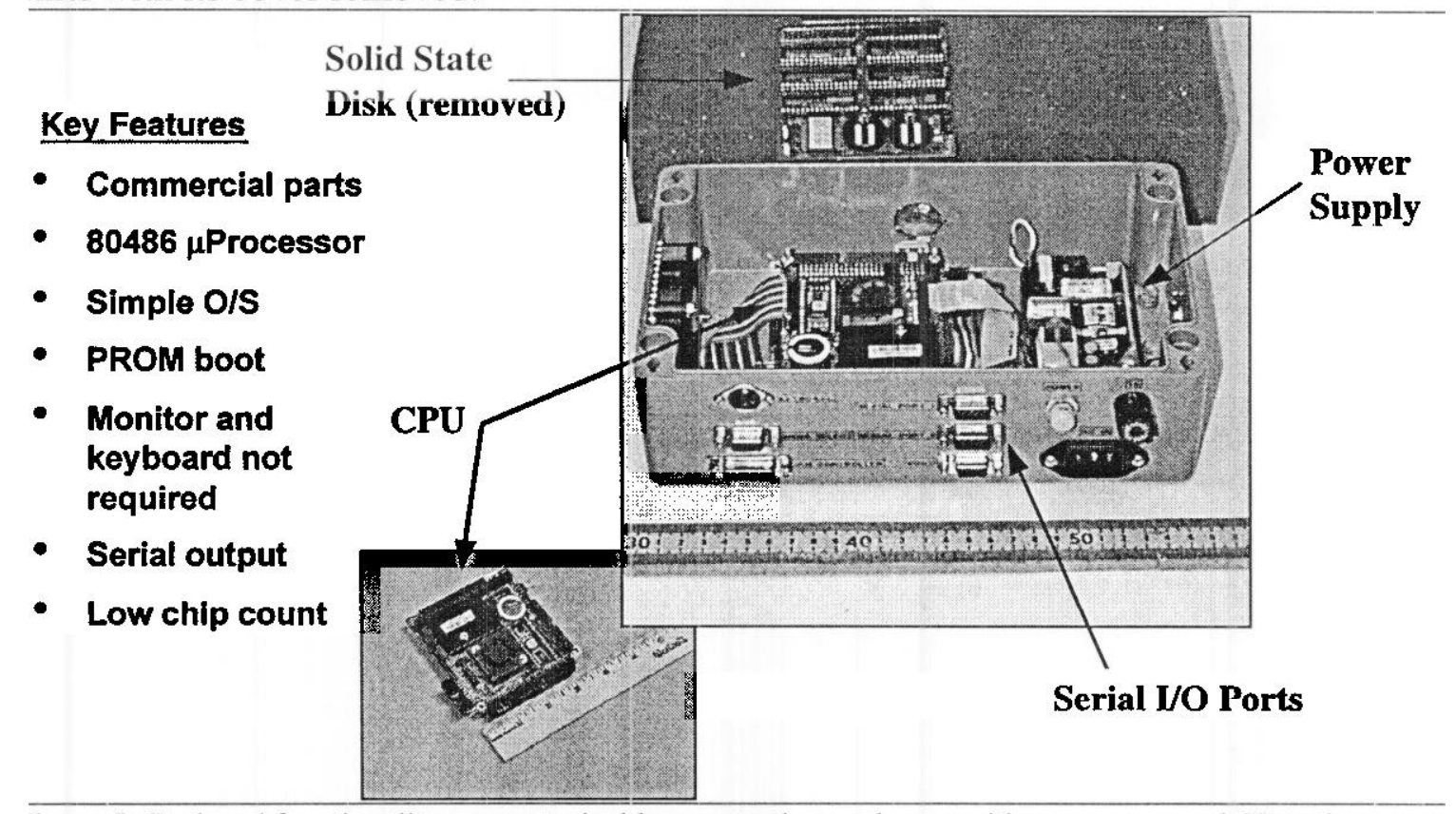

igure 5. Reduced functionality computer inside a protective enclosure with cover removed. Note the small size of the processor and memory cards. The unit shown will accommodate a keyboard and monitor for diagnostic and validation purposes. All of the data communication during normal operations takes place over the serial $\mathrm{I} / \mathrm{O}$ ports.

The computers utilize a simple commercial operating system (MS/DOS for this demonstration) which is free of the size and performance overhead of a visual user 
interface, e.g. as in Microsoft Windows and similar desktop operating systems. The computer itself contains no magnetic disks. It boots from programmable read-only memory (PROM), which also accommodates the application code. Connections exist for a keyboard and monitor, which may be used for diagnostic purposes, however they are normally not connected to the computer and the lack of them does not affect its normal operation. An additional advantage of this design is the reduced number of components present that must be inspected prior to use.

\section{Tungsten Iris}

The last of the hardware IB features present is the tungsten iris in front of the gamma-ray detector. The purpose of the iris as an information barrier is to hide geometry information and enable autonomous operation of the detection system. As Figure 6 shows, the iris assembly is attached to the front of the annular tungsten shield that is used to suppress background and interference from other sources that may be present. The iris opens and closes automatically to control the count rate such that the deadtime stays below a prescribed threshold (15\% for this demonstration.) Except for varying the effective detector solid angle, it does not affect, nor is it affected by the rest of the detection process. It utilizes a separate output line from the detector carrying the analog signal. Having the iris allows the operator to be uninvolved in metering the source distance and count time, and this is essential to establishing a measurement procedure with constant time and distance parameters for a range of possible source intensities. Since the enclosure door is closed for a classified measurement, the actual setting of the iris is invisible to the operator, and therefore the measurement geometry is unknown. This prevents witnesses from guessing at the gamma dose rate. In the event that the enclosure door is opened during a measurement, power to the iris mechanism is cut and a heavy gauge spring opens the aperture completely and immediately.

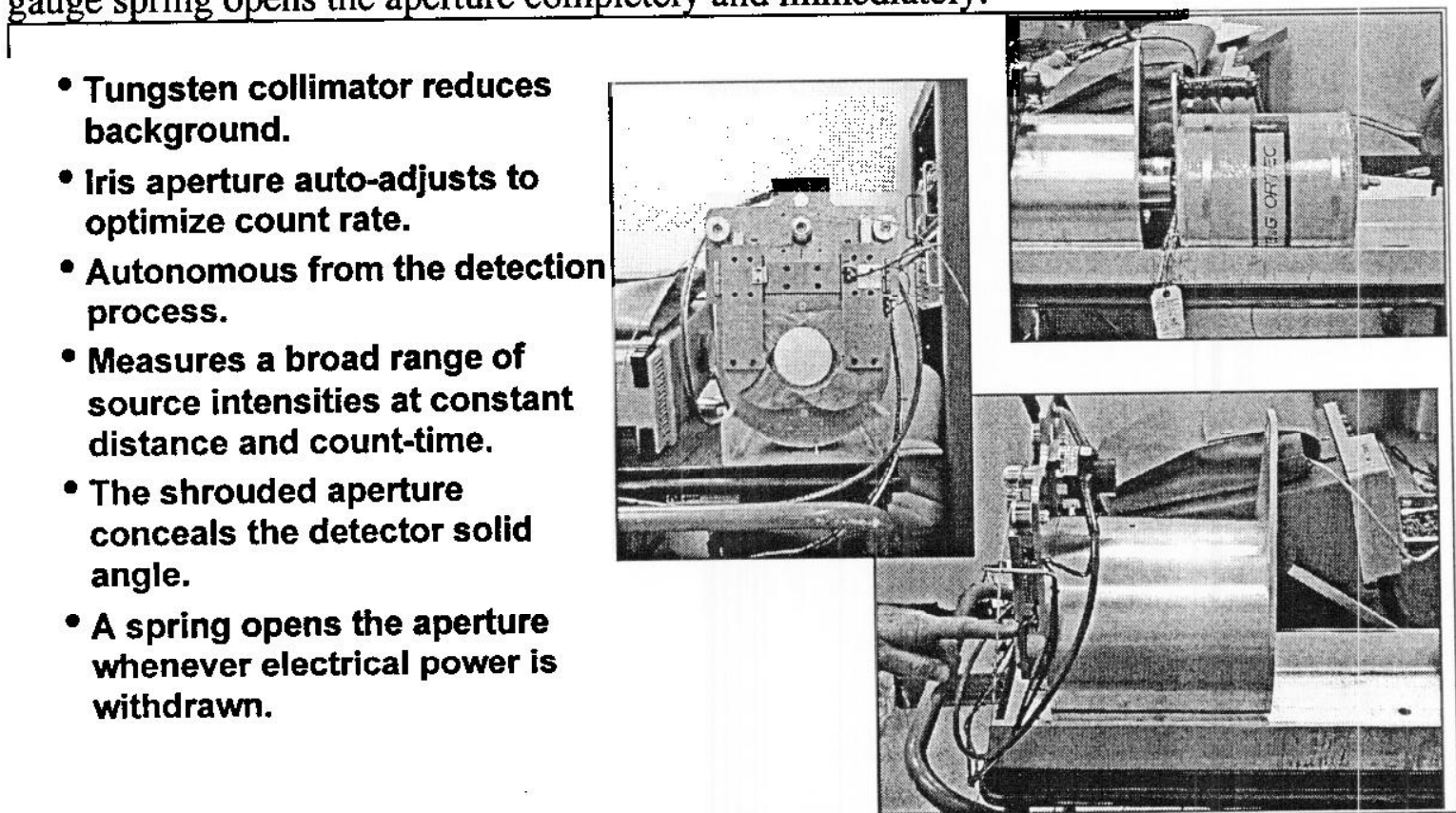

igure 6. Side and end views of the tungsten shield and iris assembly. 


\section{Conclusions}

Though it is still in the prototype stage and under development, the demonstration system meets the fundamental goals of the information barrier: it prevents classified information from being disclosed and provides reliable output. Certainly there is room for improvement. The isolation between components within enclosures can be improved as required, and tamper indications could be added. Other amendments to the hardware might make the system more inspectable. The developers are constantly considering ways to make the system simpler. One way might be to evolve away from using general purpose computers and toward more special purpose electronics. Doing so would likely sacrifice some modularity in order to gain simplicity (and presumably inspectability.) The technical staff who developed this instrument recognize their broader responsibility, not just to supply an attribute verification system that works, but one that can come to be trusted by both governments. They see this a rich opportunity for joint development. 